Citation: Nilsson, S., Shvidenko, A., Jonas, M., McCallum, I., Thomson, A. and Balzter, H. (2007): Uncertainties of a Regional Terrestrial Biota Full Carbon Account: A Systems Analysis, Water, Air and Soil Pollution: Focus, 7, 425-441, doi:

10.1007/s11267-006-9119-1

\title{
UNCERTAINTIES OF A REGIONAL TERRESTRIAL BIOTA FULL CARBON ACCOUNT: A SYSTEMS ANALYSIS
}

\author{
S. NILSSON*, A. SHVIDENKO, M. JONAS, I. McCALLUM \\ International Institute for Applied Systems Analysis, A-2361 Laxenburg, Austria
}

A. THOMSON, H. BALZTER

Center for Ecology and Hydrology, Climate and Land Surface Systems Interaction Centre, Monks Wood, UK (*author for correspondence, e-ail: nilsson@iiasa.ac.at, phone +43-2236-807-229, fax: +43-2236-807-599)

\begin{abstract}
We discuss the background and methods for estimating uncertainty in establishing a regional, terrestrial biota Full Carbon Account (FCA) in a holistic manner utilizing our experiences in generating such an account for vast regions in Northern Eurasia (at national and macro regional levels). For such an analysis, it is important to (1) provide a full account; (2) consider the relevance of a verified account taking in mind a further transition to a certified account; (3) understand that any FCA is a fuzzy system; as well as (4) understanding that a comprehensive assessment of uncertainties requires multiple harmonizing and combining system constraints of results received by different methods. An important result of this analysis is the conclusion that only a relevant integration of inventory, process based models and measurements in situ generate sufficient prerequisites for a verified FCA. We show that use of integrated methodology, at the current level of knowledge and system combination of available information, allows a verified FCA for large regions of the Northern hemisphere that could be realized for present and recent periods.
\end{abstract}

Keywords: terrestrial biota, regional full greenhouse account, uncertainty, verification, certification, Northern Eurasia

\section{Introduction}

Current knowledge of interactions between the biosphere and the atmosphere has led to the understanding that only a Full Carbon Account (itself and as the information and methodological nucleus of the Full Greenhouse Gas Account) corresponds to the sense and ultimate goals of the United Nation Framework Convention on Climate Change (Nilsson et al., 2000a; Schulze et al., 2002). Due to diverse political and economic constraints and considerations, the Kyoto Protocol and recent documents of the IPCC still operate with Partial Carbon Accounting Systems connected to the managed part of the biosphere. Recent developments process allow us to assume that the transition to full accounting will be put on the climate change science agenda in the near future. The most relevant way towards this 
development is probably through differentiation of "assessment" (i.e., it is necessary to know the real exchange of greenhouse gases between the biosphere and the atmosphere) and "accounting" (i.e. which part of this exchange is eligible to be included in the Kyoto and postKyoto accounting mechanisms). The Full Carbon Account includes two parts that differ by nature and methodology: assessing emissions caused by the anthroposphere (industry, energy, etc.) and quantifying interactions of terrestrial vegetation with other components of the biosphere and, in particular, with the atmosphere. The share of emissions of these two components in summarized fluxes of the FCA at the national level may be of the same magnitude (e.g., for Russia, Nilsson et al., 2003a). Experiences of some countries (Member States of the European Union and the USA) show that the estimated uncertainties of $\mathrm{CO}_{2}$ emissions from fuel combustion are low, as a rule, in limits of $\pm 2-4 \%$ (confidence level 0.95 ) (EEA, 2005). In spite of the higher uncertainties in other gases (e.g., in range from about \pm 17 to $\pm 48 \%$ for $\mathrm{CH}_{4}$ emissions, Monni et al., 2004; Rypdal and Winiwarter, 2001), the overall uncertainties (e.g., expressed as uncertainties in the Global Warming Potential) of the industry sectors are substantially less than uncertainties of fluxes resulting from terrestrial vegetation and agriculture (Nilsson et al., 2000a; EEA, 2005). It means that the eventual uncertainties of the Full Carbon Account mainly depend on the uncertainties generated by the biosphere, and the latter is the subject of this analysis.

The Kyoto Protocol and the following documents (IPCC, 1997, 1998, 2000, 2004a) mention the importance of assessing uncertainty, but it does not put these at the center of the problem (e.g., Nilsson et al., 2000b, 2001). For instance, the IPCC Guidelines stress that "uncertainty information is not intended to dispute the validity of the inventory estimates, but to help prioritize efforts to improve the accuracy of inventories in the future and guide decisions on methodological choice" (IPCC, 2000: p.6.5). The required reliability level of the Full Carbon Account, which should be provided at regional and global levels, is still a subject of discussion. For the partial account, which is defined by the Kyoto Protocol and subsequent international documents, Annex 1 countries have a greenhouse gas emission reduction target of 5.2\% and the European Union of $8 \%$ below the 1990 level by the first commitment period 2008-2012. It means that the uncertainties for the Full Carbon Account should be minimized to at least a level which is able to provide a reliable identification of this reduction. Some scientific discussions (e.g., in the framework of the Global Carbon Project) presumably indicate required limits of uncertainties for summarized continental carbon fluxes (expressed, e.g., as Net Biome Production) caused by terrestrial vegetation at a level of $\pm 20-25 \%$; 
evidently it seems to be too high if the Full Carbon Account were to become a subject of the post-Kyoto negotiation process. Our tentative results for temperate and boreal regions show that uncertainties of the FCA for large regions could be decreased to a level of $\sim 10-15 \%$ (confidence level 0.9), at least this level seems achievable if the FCA meets a number of system requirements and information improvements. Evidently, the technical jargon requires two clarifications. First, relative errors depend upon the estimated mean, and a definite level of uncertainties implies a tacit prerequisite that Net Biome Production (as an eventual estimate of the terrestrial biota full carbon account) has some "not insignificant" (i.e., close to zero) value. Second, strictly speaking, the completeness of the FCA cannot be estimated in any formal way, and a solution to this problem is limited by the available knowledge and level of proficiency. Nevertheless, only the philosophy of the FCA allows to develop possible approximations of such a solution.

There are two major goals of the full carbon account which are equally important and interdependent: (1) quantifying all carbon pools and fluxes included in the account; and (2) reliably estimating uncertainties. The intentions of the UNFCCC and logic of recent postKyoto developments imply the need for moving towards a verified full carbon account. A verified account means, following the IPCC Guidelines (2000: p. A3.20), that: (1) uncertainties at all stages and for all modules of the accounting scheme are estimated in a comprehensive and transparent way; and (2) the methodology of the FCA presents a guideline for how uncertainties can be managed, in particular, if the results of the accounting do not satisfy required (preliminary defined) uncertainty levels. Verification is basically a scientific notion and is (or should be) an inherent part of any accounting scheme. ${ }^{1}$ Verification which would be provided by a specially authorized independent body could lead to a certified account. Evidently, any certified account should provide a preliminary defined level of uncertainties. Currently, there is no clear understanding how certified systems could be built, in particular at continental and national scales, taking into account the many scientific, political and institutional problems that should be resolved nationally and internationally in order to do this ( $c f$. Jonas\&Nilsson, Gillenwater et al. and Jeda\&Nahorski, this issue).

\footnotetext{
${ }^{1}$ We note that Jonas and Nilsson (cf. pp. ... in this publication/book) go one terminological step further than we do here and strictly distinguish between 'validation' and 'verification' by applying science-theoretical principles. However, although we use the term 'verification' somewhat indifferently, our ultimate understanding of verification, especially in the context of our integrated (multi-measurement/modeling) approach presented here, is in line with the bottom-up/top-down accounting/verification approach discussed by Jonas and Nilsson.
} 
The way - from the current state through a verified to a certified account - is far from trivial. The Global Carbon Project (GCP, 2003) indicates, among inherent shortcomings in quantifying the carbon budget, that (1) existing global models are unable to determine carbon sources or sinks with acceptable accuracy at the regional, continental and interannual time scale, (2) there are no agreements between top-down and bottom-up approaches, (3) there are substantial inconsistencies between global and regional budgets, (4) temporal patterns are poorly understood at time scales greater than a few years, and (5) there are big gaps in comprehending the spatial and temporal pattern of human-induced fluxes.

Several methods are used in providing for the scientific basis for the terrestrial biota carbon account. A major part of the results at the continental and national levels are received from process-based models and inventory approaches. Each of these methods has well recognized strengths and weaknesses. During the last decades, process models have been advanced to explicitly incorporate current knowledge regarding ecosystem processes into model structure, and they are practically the only tool to diagnose interannual variation of major carbon fluxes. However, these models operate with a simplified, mostly "potential" world and do not have any sufficiently built system of uncertainty estimation. They allow partitioning the uncertainties in model projections caused by propagation of uncertainty in model output. But they are not able to answer the major question of any serious modeling effort: how distant is the model structure from the modeling phenomenon. Attempts at improvement of assessment of uncertainties of the process-based models (e.g., MacFarlane, 2000; Parysow, 2000; Zaehle et al., 2005) are limited by intra-model considerations, such as introduction of variability in input parameters and assessment of sensitivity of model results to this variability. On the contrary, inventory-based methods are strong by their empirical basis but they are not able to indicate rapid changes of the environment or take into account temporal trends of major drivers. Other methods used in the FCA are also very important but they serve either separate controlling blocks of the accounting system (inverse modeling) or deliver information for parametrization of the two above background methods (e.g., measurements carbon fluxes in situ).

This paper presents a brief analysis of experiences and lessons that resulted from assessing uncertainties of the terrestrial biota full carbon account (hereafter abbreviated as FCA) at the regional scale for a large region of Siberia through an EU-funded project entitled "SIBERIAII" (Multi-sensor Concepts for Greenhouse Gas Accounting of Northern Eurasia), as well as from the full carbon account of the entire Russian terrestrial vegetation carried out by 
IIASA's Forestry Program during recent years. We attempt to illustrate the fact that only a consecutive holistic approach is able to serve as the background for a verified FCA, briefly analyze systems requirements of its structure and methodology and also present typical examples ( $c f$. Jonas et al., 1999).

\section{Basic definitions}

There are many different approaches to dealing with uncertainty, and selection of the language and dimensions of the consideration is of a primary importance. We limit our analysis to informational and methodological aspects of the regional FCA leaving out consideration of different social, economic, cognitive, institutional, and ethical aspects of the problem.

The terminology used below is generally accepted in statistical theories and risk analysis. Conventional terms for standard statistical analysis are: (1) precision as reproducibility or a measure of random error; it deals with our inability to discriminate among values within a parameter, or its imprecision; (2) accuracy as correctness or a measure of the systematic error (bias) [accuracy addresses errors made in measuring within the precision of the measurement capability to discriminate]; and (3) a (rough) mistake is a measurement that is known to be incorrect due to carelessness, accident or the ineptitude of the experimenter. In a FCA, direct use of these terms is usually limited to partial and relatively simple statistical tasks, which are mostly based on direct measurements.

The mathematical theory distinguishes between uncertainty and variability. Albeit the term uncertainty is used in different meanings: statistical variability, lack of knowledge, lack of confidence in a single value (Hattis and Burmaster, 1994; Heath and Smith, 2000; Hofman and Hommonds, 1994), the use of this term in global change science is rather consistent. "Uncertainty" is understood as a description of the imperfection in knowledge of the true value of a particular quantity or its real variability in an individual (e.g., measurements of biometric indicators of trees on a sample plots) or a group (e.g., averages on sample plots established in a homogeneous category of forests). In essence, uncertainty is the absence of information; or is an expression of the degree to which a value is unknown (IPCC, 2004, 2004a; Rowe, 1994). Uncertainty can be represented by quantitative measures (e.g., a range of values calculated by various models) or by qualitative statements (e.g., reflecting the judgment of a team of experts). Variability is a special contributor to uncertainty. "Interindividual variability" means the real variation among a measured value of individuals or the parameters. In general, uncertainty is reducible by collecting additional data or by using better 
models, whereas real variability cannot be changed as a result of better or more extensive measurements (however the latter can improve the quality of estimates used). In our analysis we defined uncertainty as an aggregation of insufficiencies of our system output, regardless of whether these insufficiencies result from a lack of knowledge, the intricacies of the system or other causes ( $c f$. Nilsson et al., 2000a). Finally, uncertainties in the FCA can be expressed as confidence intervals of probability distribution functions.

Probability is the basic term of any uncertainty's assessment. The traditional approach assumes that observed frequencies are equivalent to probabilities. It requires stationary conditions of the phenomenon or process and random measurements. However, both above prerequisites are rather an exception than the rule in a FGA. In addition, fuzziness of the FCA inevitably leads to the use of subjective (personal) probabilities, specifics of which (applicably to the FCA) we consider below.

\section{Uncertainties of the regional full carbon account}

Strictly speaking the "ideal" FCA should be a result of the continuous monitoring of terrestrial biota in space and time. The background philosophy of such monitoring leads to the idea of an integrated observing system and further - to an integrated accounting system. Recent developments allow us to conclude that some simplified patterns of such an approach could be realized in the near future. Currently, all carbon accounting schemes are forced to use many heterogeneous information sources including results from different measurements, assessments, as well as expert estimates over time. This procedure generates numerous and diverse uncertainties. Taking into account methodological specifics of the carbon account, different classifications (decomposing, categorizing) of uncertainties can be relevant. For the IPCC TAR Assessment, Moss and Schneider (2000) considered four major groups dealing with (1) confidence in the theory, (2) observations (measurements), (3) models, and (4) consensus within a discipline. Rowe (1994) considering common aspects of risk analysis divided uncertainties into temporal (past and future), structural (complexity), metric (measurements), and translational (explaining uncertain results). Distinguishing two broad classes of uncertainty - "statistical" (associated with parameter or observational value that are not known precisely) and "structural" (referring to causal relationships between variables) the IPCC Workshop on Describing Scientific Uncertainties in Climate Change pointed out substantial difficulties to assess structural uncertainty and limited possibilities to do it in any comprehensive formal way (IPCC, 2004a). 
For structuring the FCA uncertainty calculation schemes, a more detailed classification of uncertainty's sources in the following groups seems useful (see also Shvidenko et al., 1996; Jonas et al., 1999; Nilsson et al., 2000; ).

1) Definitions and classification schemes used in calculations. As a rule, the definitions and classification schemes currently used in the FCA have been introduced for purposes other than carbon accounting, and often correspond to inappropriate and obsolete standards and measuring technologies.

2) Shortcomings of available data. Some important data have never been and are not being measured, which leads to incomplete and sometimes inappropriate substitutions.

3) Unknown or insufficient precision of measured data. Reasons for this could vary: subjective (not random) sampling; biased statistics; deliberate falsification; inappropriate measurement techniques; etc.).

4) Lack of a proper basis for upscaling. Very often, there is no solid platform for estimating the accuracy of upscaled point measurements, gradients are unknown and stratification is provided based on expert judgments.

5) Short time series. Some processes require historical reconstruction for up to 150-200 years which is not covered by existing historical records.

6) Lack of knowledge of some important processes. For instance, the post-disturbance processes in soil on permafrost, some aspects of below-ground NPP, or nitrogen turnover after biotic disturbances are to a significant extent a "black box".

7) Oversimplification of the modeling approach. In both major methodological approaches of the carbon account, i.e. pool based and flux-based carbon account, the regional Full Carbon Budget (FCB) is presented by a sophisticated superimposition of (almost exclusively) nonstationary stochastic processes. There is still no methodology which would use this intrinsic feature of an FCB as a prerequisite for its modeling and quantification, and the substitution of stochastic processes with deterministic models is common practice. There are many other examples of this type.

8) Spatially and/or temporarily insufficient observing systems. Significant remote areas (e.g., in the Russian north) are not covered by high quality remote sensing observations (due to low sun angle and boreal winter night) or on-ground observations. Some indicators are very 
dynamic, and existing monitoring systems and available data cannot grasp these dynamics, e.g., seasonal dynamics of insect outbreaks in boreal forests.

Although each class of uncertainties can be addressed separately, they are not necessarily independent, and their interdependence should be examined. The above list of uncertainty sources can be applied to some or all periods of the assessment: past, present and future. However, any prediction and forecast includes the need to consider many other uncertainties dealing with future drivers (climatic, ecological, social and economic), and responses and feedbacks of terrestrial ecosystems. The level of background uncertainties could be illustrated by the uncertainties of climatic predictions. Using 12 three-dimensional general circulation models (GCMs), including seasonal cycles, a mixed layer ocean and interactive clouds and other features, the projected increase in global mean surface air temperature under equilibrium conditions for doubled $\mathrm{CO}_{2}$ concentrations in the atmosphere varies approximately three-fold (from 1.6 to $5.4^{\circ} \mathrm{C}$, mean $3.82^{\circ} \mathrm{C}$, coefficient of variation 26.3\%) (Cess et al., 1993). In spite of evident progress in climatic modeling during the last decade, the situation has not changed significantly (e.g., Collins et al. 2005). One can conclude that no solid background of a verified FCA for future periods of time exists. We will not consider this special (and highly uncertain) case further.

Considering the sense, information and methodological limitations of learning the terrestrial biota full carbon budget, we can conclude that any FCA is a typical fuzzy system. In spite of thousands of publications since Zadeh (1965) has published his fundamental paper, there is not a single unique definition of fuzzy systems and/or fuzzyness. We use this term in its rather common but wide mathematical sense (Kosko, 1994; Wang and Barret, 2003) bearing in mind that many elements of the FCB (procedures, components and stages of the FCA) do not present a crisp set, but require the knowledge of multi-valued membership functions. In essence, "fuzzy logic is part of a formal mathematical theory for the representation of uncertain systems" (Cogan, 2001), and "the concept has generally been associated with complexity, vagueness, ambiguity, and imprecision" that "further implies that model coefficients, parameters, or functional relationships may be fuzzy and, hence, not known with complete certainty" (Mendoza and Sprouse, 1989). The comprehensive development of the formal theory, which would provide for learning about natural fuzzy systems is, to a significant extent, a matter for the future. Although fuzzy logic and fuzzy methods are recommended as a means to incorporate subjective information in different aspects of assessing uncertainties (e.g., Haimes et al., 1994; Hattis and Burmaster, 1994), their 
applications in ecology and natural management are limited by numerous and diverse but partial tasks (Mendoza and Sprouse, 1989; Bare and Mendoza, 1991; Wan-Xiong et al., 2003; Chen and Mynett, 2003; Özesmi and Özesmi, 2004 etc.). In the framework of FCA, it is productive to apply "fuzzy thinking", a philosophical approach, which helps much in structuring problems, developing a relevant FCA system and treating uncertainties. Elements of this approach little-by-little are introduced in different parts of global change science. During the last years, this philosophy has also been applied to a "multiple-constraint" approach, where heterogeneous data - measurements of fluxes, remote sensing data, data from different inventories, etc. - provide constraints in models used and in assessing results (e.g., Wang and Barret, 2003). Evidently, "fuzzy thinking" can include a formal definition of membership functions and inference rules, but it is not exhausted by exclusive applications of fuzzy logic methods.

"Fuzzy thinking" leads to an important conclusion which defines a relevant specific methodology of the verified FCA: strictly speaking, any individual method or model of the FCA applied separately is unable to provide a sufficient (i.e., comprehensive, transparent and reliable) estimation of uncertainties. It defines the need to systematically integrate relevant methods and models. It leads to the philosophy of integration in all its ramifications. For the FCA, the solution is an integration of all relevant information sources (on-ground, remote sensing data and appropriate regional ecological models), soft and hard knowledge. On the other hand, integration should be provided for different components of the FCA: carbon of terrestrial biota, ocean and atmosphere. A consistency of the terrestrial biota global carbon budget is an indicator of its reliability. Comparing the results received by different methods is an important part of verification.

An additional dimension of uncertainty is generated by the requirement of having a full carbon account. By definition, "a full $\mathrm{C}$ budget encompasses all components of all ecosystems and is applied continuously in time" (Steffen et al., 1998). However, in spite of progress over the last decade, there remain substantial uncertainties in understanding regional and global carbon budgets. This permits estimating the completeness of the FCA only by expert judgment ${ }^{2}$. The judgment of the completeness of estimating an FCB continuously in time can be also satisfied in a very approximate manner. Because the FCB has a "memory", up-to-date estimates of $\mathrm{C}$ fluxes may strongly depend upon the previous, sometimes long periods for

\footnotetext{
${ }^{2}$ We distinguish between a full carbon budget (FCB) as a natural system, and a full carbon account (FCA) as an artificial accounting system.
} 
which relevant measurements may not be provided, and required information simply does not exist. In addition, the completeness greatly depends upon the end-point user target. For example, the final goal of carbon accounts can be defined either as an assessment of the amount of $\mathrm{C}-\mathrm{CO}_{2}$ in the exchange, or quantities of all carbon contained gases, or the Global Warming Potential. Nevertheless, experiences of the FCA for some countries (like Russia and Austria) show that about 96-98\% of recognized carbon fluxes are usually included in the consideration, although in essence this conclusion is an expert estimate (Nilsson et al., 2000a). The completeness allows us to implement a balance estimation and an analysis of the consistency of individual modules and blocks of the FCA. Here we face a substantial methodological shortcoming of any Partial Accounting System: the lack of possibility for both closing the balance and checking consistency of the accounting system. The crucial assumption underlying partial carbon account is that some drivers and, consequently, some net carbon fluxes (especially those that are not directly human-induced) are untested, and their changes remain unknown. Thus, the FCA presents additional information for estimating (final) uncertainties of the accounting systems, as well as for understanding the specifics, strengths and weaknesses of partial accounting systems.

\section{Requirements to the Terrestrial Biota Regional Full Carbon Account}

The following important requirements to any verified FCA result from the above considerations.

1. Only a holistic system approach (with modifications resulting from the fuzziness of the FCA) can serve as a solid overall methodological background of the FCA. From a substantial point of view, implementation of the landscape-ecosystem methodology is one of only a few possibilities for a consecutive system analysis. The landscape-ecosystem approach postulates that an ecosystem (i.e., vegetation-soil ensemble of different scale) is considered as the primary unit of scientific description, modeling and interpretation, and the quantification of intra-ecosystem processes of energy and matter exchange should include the impacts of properties of an individual landscape. From an informational point of view, all relevant sources of information have to be used including as comprehensive as possible ground-based quantitative descriptions of ecosystems and landscapes (e.g., in the form of Geographic Information Systems), remote sensing data, numerous and diverse sets of auxiliary models (e.g., for connecting remotely sensed data with "hidden" ecological parameters of ecosystems), measurements of fluxes (such as Net Ecosystem 
Exchange) and concentrations of gas composition in the atmosphere, and regional ecological models of different types. From a methodological point of view, a relevant combination of pool-based and flux-based approaches allows to eliminate (to a possible extent) weaknesses of each of these basic methods of the FCA.

2. Use of strict and mono-semantic definitions and formally complete classification schemes. This problem is not trivial. Recent activities of the FAO on harmonizing forest-related definitions for use by various stakeholders present many examples on how many different problems could be met only in the rather simple field of land use - land cover classifications (FAO, 2002-2005).

3. Explicit structuring of the account; use of strict intra-system (module) spatial, temporal and process boundaries. With this respect, a number of questions should be regulated, e.g., whether human consumption of vegetation products should be considered as part of NBP, etc.

4. Estimation of uncertainties should be provided at all stages and for all modules of the FCA. In particular it allows gaining additional information needed for understanding relevant ways for the management of uncertainties.

5. Accounting schemes, models, and assumptions should be presented in an explicit algorithmic form. It means that the use of soft knowledge (e.g., in the form of expert estimates), which is inevitable in the FCA, should be provided in a "quantified" form and by methods which would allow to minimize shortcomings and possible bias of subjective information.

6.The accounting scheme should provide a spatially explicit distribution of considered pools and fluxes. It means that all major components of the FCA should be georeferenced at relevant scales.

7. Temporal dimensions of the FCA should be consistent with the temporal peculiarities of processes which are quantified (modeled). The relevant length of respective time scales and the required frequency of observations are defined by specifics of the individual processes considered. Obviously, a year or a different period of accounting should be clearly identified.

Some of the above requirements are not satisfied in regional and national accounting and are not indicated in the recommendations of the IPCC (IPCC, 1997; IPCC, 2000). This increases the fuzziness and the role of expert components in the FCA. 


\section{Assessing uncertainties}

Two main statistical tools - probability density functions and confidence limits - are normally used for assessing uncertainties. The IPCC Guidelines suggest the use of a 95\% confidence interval. This "conventional" recommendation is usually explained by simplicity of calculation of the interval corresponding to two standard errors. It is not completely clear, however, how much this traditional recommendation (1) corresponds to the specifics of the FCA and (2) impacts interdependence of type I (alpha) and II (beta) errors. Considerations which are beyond these comments are as follows. In essence, selection of a confidence interval should be based on a function of losses due to an achieved level of uncertainties. However, there are both no developed formal theory for quantification of such a function and big practical difficulties in its structuring. Thus, solution of this question remains in the field of expert estimates and should be a result of substantial analysis and, finally, of an agreement of interested parties. Taking into account the sense of the FCA for large territories and practical consequences of its uncertainties, one could conclude that the relevant confidential interval (e.g., for NBP) should correspond to probability smaller than 0.95 (e.g. in the range of 0.8-0.9), or even smaller. Besides, it would allow to decrease to a relevant value of errors of type II. This problem, due to our knowledge, has not been considered in available practical assessment of uncertainties of the FCA. From another side, the numerical expression of uncertainties (i.e., statements of the type: the uncertainty (accuracy) of the final result is at $p$ percent) has a substantial psychological meaning, at least for the public and policy makers. Uncertainties of some important components of the carbon budget of terrestrial vegetation are high and - if we use a confidential interval for high probability - could be comparable with, or even exceed, $100 \%$. Evidently, any results with uncertainties $>100 \%$ have no practical meaning. Thus, artificial setting of high confidential intervals can generate a wrong impression about the practical applicability of final results of the FCA. This problem requires further elaboration. In examples and considerations below we use the confidence level of 0.9. We examined the following method of assessing uncertainties in the FCA: (1) estimation of precision of all intermediate and final results; (2) "transformation" of precision into uncertainty; and (3) multiple-constraint comparisons of results.

Estimation of precision. The FCA is presented as a hierarchical structure of analytical expressions. It allows the formal use of error propagation theory assuming that variables used in the calculations are approximately normally distributed. However, only some of the initial data result from direct measurements for which standard errors, probability distribution 
functions etc. can be estimated with conventional statistical methods. This generates some open issues: (1) the need to use estimates of precision of initial variables "by analogy" (i.e., average values by classes of the classification used), or based on expert estimates and subjective probabilities, and (2) the use of "summarized errors" as a substitute of random errors. As a rule, it is impossible to divide random and systematic errors of many initial variables used in the FCA. Thus, summarized errors are considered as some functions of random and systematic errors. In practical situations, the share of bias is relatively small (estimated in the order of $10-15 \%$ of the random error). In such cases, applications of the error propagation theory do not change the essence of statistical conclusions.

"Transformation" of precision into uncertainties. The calculated precision is transformed into uncertainty based on sensitivity analysis and expert estimates of unaccounted impacts and processes. The Monte Carlo method is often used as a tool for sensitivity analysis. Details of this procedure depend upon the end-point target of the assessment. (1) The endpoint is a fixed but unknown value (e.g., Net Biome Production). Values are sampled at random from distributions representing various "degrees of belief" about the unknown "fixed" values of the parameters (i.e., the true but unknown value is equal to or less than any value selected from distribution). The subjective confidence statement about the true but unknown assessment end point accounts for multiple sources of uncertainties (inventory or model structure; presence, variability, and representatives of data; quantified expert opinions, etc.). Uncertainty about a quantity that is fixed (or deterministic) with respect to the assessment end point is often called Type B uncertainty. Variation of input data allows the selection of "important input parameters" which contribute most to the spread in the distribution of the FCA results. (2) The end point is an unknown distribution of values. In such a case, the Monte Carlo simulations are performed in two dimensions producing numerous alternative representations of the true but unknown distributions (assessment of uncertainty of Type A). In practical applications of the FCA, both of the above procedures are used, however it often occurs that a mixture of both types of uncertainties is presented.

Although Monte Carlo calculations are not free from some subjective elements (e.g., a "selection" of the type of the unknown distribution), this method presents both comprehensive information about uncertainties of the accounting scheme (model) and important information for management of uncertainties. These results often serve as an iterative step in a process to improve model estimates. 
However, we have to note that all of these results are true only inside of the approach (model) used, under given inputs and assumptions, and can have little in common with reality, if the model or assumptions are not "comprehensive" or oversimplified. Thus, if, e.g., model FORCARB (carbon inventory for 2000 for private timberland of USA, which covers about $75 \%$ of the country's productive forests) estimates uncertainty $\pm 9 \%$ of the estimated median of the total carbon in the year 2000 and $\pm 11 \%$ in the projection year 2040 (Heath and Smith, 2000) - this is only an information that Monte Carlo calculations have presented these results using the above model (which is rather simple), but nothing about a "real uncertainty". We are far from any intention to criticize this particular model - this simply serves as an example to demonstrate specifics which are inherent to any, even very complicated model. It explains the need for independent, thorough analysis of the completeness and structural rationality of the FCA that is used. One way to provide this analysis is by using experts' judgments on the topic; these judgments are quantified and embedded (in addition to Monte Carlo or other methods of sensitivity analysis) into final values of uncertainties (Shvidenko and Nilsson, 2003).

Multiple-constraint comparison of results. The balance and consistency analysis of carbon budgets of relatively closed blocks (modules) of the FCA, comparisons of independently calculated intermediate results and multi-constraints analysis of final results - three important techniques, which allow us to make a final judgment about the FCA. We have to point out the crucial importance of the multiple-constraint methodology. The "top-down - bottom-up" analysis is currently a major tool for understanding the "real" range of uncertainties of the global carbon budget (see Jonas and Nilsson, this issue). This could be very useful at continental and other macro-regional FCAs. Hence, the FCA for Russia showed that the former intensively debated problem of the missing sink is a result of the incompleteness of the account (Nilsson et al., 2003).

The problem of bias. A usual prerequisite of uncertainty analysis is that approaches used do not generate significant bias. As a rule, this assumption is very difficult to check in practical assessments. Bias is often caused by temporal or spatial non-stationarity of processes or ways in which measurements are provided. Improvements of measurement techniques or methodologies used, as well as new knowledge could generate a substantial shift in results, indicating previously unrecognized biases. We present two recent examples which illustrate the magnitude of the possible impacts. 
(1) The first detailed inventory estimate of the NPP of Russian forests for 1993 was based on a database which contained approximately 3000 sample plots where measurements were performed by traditional destructive sampling (Nilsson et al., 2000). These measurements did not account for some important components of NPP (e.g., root exudates which comprise about $15 \%$ of the total NPP of boreal forest ecosystems) and probably underestimated NPP of fine roots. The transition to a semi-empirical inventory-based modeling system which does not have significant recognized biases (at the current level of understanding), has increased the average forest NPP in Russia by approximately one-third (Shvidenko et al., 2006). Tendencies of the same magnitude have been also recognized for NPP of wetlands in Siberia (Vasiliev et al., 2001).

(2) Based on remotely sensed NDVI, Myneni et al. (2001) have estimated the sequestration of carbon in above ground wood of Russian forests at $283 \mathrm{Tg} \mathrm{C} \mathrm{yr}^{-1}$ for the period of 1992-1998. This accumulation corresponds to the increase of growing stock volume at about 1 billion $\mathrm{m}^{3}$ annually. The forest inventory data for the same period indicate the increase of growing stock at almost 3 times less (Shvidenko and Nilsson, 2003). This contradiction has been recently explained (Lapenis et al., 2005). The recent analysis of temporal dynamics of allometric ratios of different phytomass fractions during the last 50 years has recognized the substantially different trends of above ground wood, green parts and roots. The calibration procedure provided by Myneni et al. did not take into account these dynamics. If the latest is included, the remote sensing estimate is decreasing to the level compatible with the forest inventory data.

\section{Some practical implementations and results from case studies}

We attempted to introduce (to the extent possible) the above requirements and techniques in estimating the FCA for two regions with different conditions: (1) Russia as a whole country, and (2) a large ( $\sim 3$ million $\mathrm{km}^{2}$ ) region of Northern Eurasia (SIBERIA-II study area, see Box 2). In spite of both different availability of information and appropriate levels of details, the methodology of the FCA for these two objects had many common features. The information base has been developed in the form of an Integrated Land Information System (ILIS) which is comprised of a multi-layer GIS and corresponding attribute data (at scale 1:2.5 million for the entire country and 1:1 million for the SIBERIA-II region). All relevant information sources have been used for the development of the ILIS: available maps and legends; data from different inventories (in particular, forest inventory) and surveys; various scientific archives; official statistical data; etc. The landscape-ecosystem methodology served as the 
overall scientific basis of the account which was based on an integration of pool-based and flux-based approaches. The flux-based approach is expressed as assessing fluxes (measured in

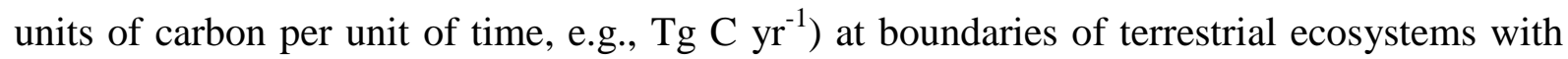
other components of the biosphere (atmosphere, lithosphere, hydrosphere)

$$
\mathrm{NBP}=\mathrm{NPP}-\mathrm{HSR}-\mathrm{DEC}-\mathrm{D}-\mathrm{TL}-\mathrm{TH},
$$

where NBP and NPP are net Biome and Net Primary Production, HSR - heterotrophic soil respiration, DEC - flux due to decomposition of coarse woody debris, D - flux due to disturbances, TL and TH - fluxes to lithosphere and hydrosphere. The pool-based method estimates carbon pools at the beginning and end of the assessment period. A combination of these two approaches (or - in an ideal case - comparison of independently received results) results allows us to estimate the methodological consistency of the FCA.

We present some typical examples from the above two case studies. For the whole country we provided our estimation of the FCA for the initial period of the Kyoto Protocol (1988-1992). We have to note that the terminology of the Protocol ("since 1990") is not completely appropriate for any solid carbon budget estimation at the national level independently whether full or partial carbon account is considered: information required for the FCA of large territories cannot be made operational at the yearly time-scale. Thus, the estimation of uncertainties was provided for 5 year averages (1988-2002).

Two major conclusions follow from the FCA for terrestrial biota of all Russia: (1) resulting uncertainties in the FCA are relatively high: the Net Biome Production (including human consumption of vegetation products) was estimated as $0.35 \pm 0.18 \mathrm{Pg} \mathrm{C} \mathrm{yr}^{-1}$; (2) the greatest uncertainty lies in assessing soil processes (the change of soil organic carbon was estimated

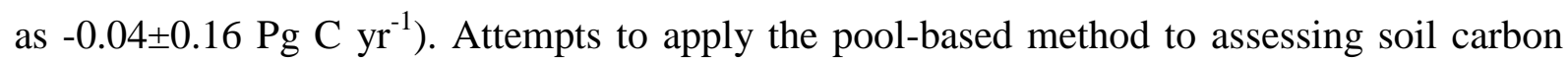
dynamics were insufficient due to the lack of required information. It has been shown, however, that principal improvements of reliability of results are possible only in the framework of the full account, and that the problem of the "missing sink" is the problem of incompleteness of assessments (Nilsson et al., 2003). The overall methodological lesson from this study was that any crucial decrease of uncertainties of the FCA at the national level requires substantial improvements of input information which probably could be sufficiently done in the framework of integrated observing systems like GEOSS (Global Earth Observation System of Systems). Comprehensive use of (only) existing information could supply satisfactory results in assessing relatively simple components of the FCA, although 
even in such cases substantial expert elements remain (see Box 1 for assessing uncertainties of forest phytomass as an example).

\section{Box1. Uncertainties of estimation of the total amount of phytomass in Russian forests based on data of forest inventory}

Initial assumptions: Data of the State Forest Account (SFA, i.e., aggregated data of forest inventory by $\sim 2000$ forest enterprises) and regression equations of phytomass do not have any bias at an accepted level of significance. In order to check these assumptions, a special statistical and expert analysis of data and procedures have been provided.

Indexes used: $i$ is phytomass fraction, $i=1, \ldots, 7 ; \rho$ is dominant species, $\rho=1, \ldots, 27 ; m$ is ecoregion, $m=1, \ldots, 141, k-$ number of forest stands.

Variables: $M$ - mass (dry matter) of fractions, Tg; GS - growing stock volume, $\mathrm{m}^{3} ; A, S I, R S$ - age, site index, and relative stocking, respectively; $\delta$ - content of carbon in phytomass.

Initial data are presented by a matrix for each 141 ecoregions over the country, which contains area and growing stock distributed by age classes $A$ for dominant species $\rho$ and types of inventory $r(r=1,2,3)$, as well as average $S I$ and $R S$ by species and inventory types.

Mass $M$ of fraction $i$, dominant species $\rho$, ecoregion $m$ is calculated as

$$
M_{i r m}=d_{i} \stackrel{a}{a}_{A=1}^{q} R_{i r m A} \ngtr G S_{r m A}=d_{i} \stackrel{a}{a}_{A=1}^{q} c_{0} S I^{c 1} A^{c 2+c 3 R S+c 4 R S 2}
$$

where $R$ is ratio of phytomass fraction to growing stock (expressed as multi dimensional regression of $A, S I$ and $R \mathrm{~S}$ ) and $c_{0}, \ldots, c_{4}$ are regression coefficients. Thus, the total phytomass of Russian forests is

$$
M=\stackrel{141}{\stackrel{\circ}{\mathrm{a}}} \underset{m=1}{\stackrel{2}{\mathrm{a}}} \underset{r=1}{\stackrel{\mathrm{a}}{7}} M_{i=1}
$$

Based on standard methods of error propagation theory, the summarized error of (B1.2) could be expressed in an explicit way (Nilsson et al., 2000a). Applying the set of equations for $\mathrm{R}_{\mathrm{ipm}}$ (Shvidenko et al., 2002), we have got the summarized error of forest phytomass by ecoregion in the range of $\pm 5-14 \%$ (here and below the confidence level is 0.9 ) and the final precision (weighted by total ecoregion's mass of phytomass) is estimated at about $\pm 3 \%$. Assuming the relative error of $\delta_{i}= \pm 2 \%$, we come to the final conclusion that the total summarized error is 
$\pm 3.7 \%$, and the confidence interval is $32.9 \pm 1.2 \mathrm{Pg} \mathrm{C}$. This result represents a formal estimate of precision. In order to assess how much expert estimates and assumptions used were able to impact this conclusion, 5 Russian experts were requested to estimate the completeness of accounting. The conclusion was consistent that the assessment has accounted for "not less than $2 / 3$ of all uncertainties", i.e. the final uncertainty was estimated to be about $\pm 4.5 \%$. Additional information can be presented by comparison with independent estimates from other sources. However, from nine different estimates of forest phytomass in Russia reported during the last two decades we were able to select only four which used sufficient information and accepted methodologies. The average densities for forest phytomass in Russia from these sources were: Alexeyev and Birdsey (1994) - $3.63 \mathrm{~kg} \mathrm{C} \mathrm{m}^{-2}$, Isaev et al. (1995) - $4.55 \mathrm{~kg} \mathrm{C}$ $\mathrm{m}^{-2}$, Isaev and Korovin (1998) $4.51 \mathrm{~kg} \mathrm{C} \mathrm{m}^{-2}$, and IIASA (independent GIS-based method, Nilsson et al., 2000) $-4.403 \mathrm{~kg} \mathrm{C} \mathrm{m}^{-2}$. The average of these estimates is $4.27 \mathrm{~kg} \mathrm{C} \mathrm{m}^{-2}$, or $0.7 \%$ to our estimates of $4.30 \mathrm{~kg} \mathrm{C} \mathrm{m}^{-2}$.

Nevertheless, the following considerations illustrate some "hidden" uncertainties, which cannot be recognized by any formal analysis. The estimate of $4.30 \mathrm{~kg} \mathrm{C} \mathrm{m}^{-2}$ has been received based on a set of models which had been developed using experimental data available in 1997. The models have been recalculated with use of additional experimental data accumulated in 1997-2004 (the number of sample plots has been increased by about $10 \%$ to about 3600). The new set of the models has been applied to the same data of the SFA-1993, and a new estimate of the density was $4.43 \mathrm{~kg} \mathrm{C} \mathrm{m}^{-2}$, or of about $3 \%$ more than the previous one. Evidently, this is within probabilistic limits of the uncertainty estimated above. Finally, the assumption that the SFA growing stock has no bias is not true: an estimate of the bias for 1993 is $+2.5 \%$ (Shvidenko and Nilsson, 2002). However, 59\% of Russian forests (by growing stock) were composed of mature, overmature, and uneven-aged forests which have a substantial amount of trunk and root decay. An approximate conservative estimate gives the values between 2 and $3 \%$ of the growing stock, i.e. we have an approximate compensation of the bias of the growing stock estimation. Thus, the overall conclusion is that uncertainties of our knowledge of phytomass of Russian forest ecosystems in 1993 are at the level of 5-6\% with high probability (not less than 0.9 ).

\section{End of Box 1}

SIBERIA-II aims at the full greenhouse gas account based on a fusion of multi-sensor remote sensing concept, comprehensive description of individual ecosystems and landscapes in the form of an ILIS and use of ecological models of different types. A number of specific features 
of SIBERIA-II allowed to substantially increase reliability of the regional FCA. First, an introduction of multi-sensor remote sensing (RS) concept has substantially increased quality and efficiency of information. Considering the large scale and remoteness of the region, information presented by RS ( 12 different sensors were examined) was of crucial importance for updating land cover, estimating disturbances, assessing some environmental indicators, etc. However, many inconsistencies in the technical capacities of RS sensors, spatial and temporal resolution and needs of the FCA have been recognized. Needs of new technical RS tools which would be specially designated for studying the biospheric role of terrestrial biota are evident. A good example of this type is the need of a satellite with P-band radar on board for assessing vegetation (particularly forest) above ground biomass. Second, the objective in using diverse information was to increase the synergy from combining various, relevant information sources. Third, an application of different ecological models presented the possibility of multiple constraints of the results as well as the possibility of independent estimates of many components of the FCA.

The examples presented below are typical. They are limited by the approach which is based on the ecosystem-landscape methodology. A GIS-layer "relevant for the FCA" and corresponding DB were developed at the polygon level. The FCA is provided for each of the polygons (which serve as a primary ecosystem-landscape unit) and is aggregated by ecoregions. Part of the components of the FCA is estimated based on regional ecosystemlandscape models. It puts special requirements on the hierarchical structure of the classification of land classes used in order to limit the variability of the FCA components within the classes. From a modeling point of view, the approach consecutively examines three FCA varieties: (1) "base-line" inventory, assessing average values, (2) introduction of the latter environmental indicators by using empirical and semi-empirical ecosystem and landscape models, (3) use of process-based blocks as part of the multiple-constraints procedure.

The following major lessons learned from this regional case-study seem important.

1) It has supported the appropriateness of an ecosystem-landscape approach as the scientific background for the regional FCA.

2) Vegetation components of the FCA for individual polygons are estimated with high reliability. Hence, live biomass (phytomass) by polygons is defined with uncertainties $\pm 7-$ 15\%, Net Primary Production and Heterotrophic Soil Respiration $\pm 15-20 \%$ (confidential probability here and below 0.9 ). However, it has required the development of a number of 
special regional modeling systems based on a big amount of sample plots (between several hundred and several thousand for each component) and the use of all available reference and normative information (yield tables, models of gross and net growth, etc.).

3) The uncertainty of estimates of soil carbon pools is high (in range of $\pm 10-15 \%$ ) and contains a substantial share of expert elements and assumptions due to the coarse resolution of soil data (the basic soil map and reference DBs are presented at a scale of 1:1 million), obsolete and unevenly distributed measurements, mapping at different time scales, and insufficiently documented history of vegetation fire during the two recent decades. At the ecoregion level uncertainties of major pools and fluxes (like NPP, HSR) are estimated to be in the range of 5-10\% (each ecoregion contains 600 to 4000 polygons), under the assumption that the account has no significant bias (more information and typical examples are given in Box 2). Calculations provided by both pool-based and flux-based methods showed rather consistent results, although assessing the soil carbon dynamic is substantially less certain than for other carbon pools (phytomass, coarse woody debris).

4) Some problems with estimating uncertainties are generated by aggregation of ecosystems in polygons taking into account the coarse scale of the accounting. To some extent these uncertainties are decreased by the implementation of "mixed classes" (e.g., polygons, which contain more than one class). On the other hand, implementation of "virtual polygons" presents the additional possibility to decrease uncertainties of this type. "Virtual polygons" comprises land classes, which are represented by numerous plots of small areas and are not individually indicated at the GIS layer (roads, small rivers and water reservoirs, settlements, some classes of agricultural lands, etc). As a rule, the total area of such land classes could be received from independent sources, and corresponding corrections of an area are provided at the ecoregion level. However, the aggregation is substantially based on professional judgments, and estimating these uncertainties includes substantial expert components.

5) Interannual variability of the FCA could be very high (up to 2-5 fold for NBP and up to $25-30 \%$ for NPP during a 10-15 year period) and is defined by the impacts of seasonal weather specifics and by the extent and severity of disturbances.

(6) Uncertainties of an FCA estimated for an individual year could be very high. Thus, considering time series is the best strategy for reducing uncertainty.

\section{Box 2. Monte Carlo estimation of uncertainties of phytomass, NPP and NEP at the regional level (SIBERIA-II) for a base year 2003.}


The region of SIBERIA-II, of the total area of 307.8 million ha stretches for about 3,000 km from the Arctic ocean to the boundary with the Tuva Republic in the south and includes major vegetation zones of the Northern Hemisphere (polar desert, tundra, forest tundra, northern, sparse, middle and southern taiga, temperate forests, forest steppe, steppe and semi-desert). The area of the region is divided in 23 ecological regions (ecoregions) and $\sim 35,000$ polygons of which 16,589 are covered by vegetation (ecosystem-landscape units). The FCA was provided by polygon. Phytomass by 7 fractions was estimated as described in Box 1. Net Primary Production (NPP) was calculated based on a special method of modeling of the annual cycle of Total Production of Phytomass (TPPh). The method, algorithm and parameterization used are described in Shvidenko et al. (2004). Estimation of the FCA was provided similarly to equation (1) with some technical modification. Monte Carlo simulations (15,000 runs per simulation) have been provided for phytomass by fractions, NPP and NEP at both polygon and ecoregion levels. Input uncertainties for simulation have been estimated as follows: growing stock $\pm 15-20 \%$ (requirements of Forest Inventory Manual addressed to separate stand are $\pm 12-15 \%$ ), site index $\pm 5 \%$, age \pm 10 -40 years dependent upon average age of stand and dominant species, relative stocking $\pm 15-20 \%$. Below we present some results of the simulations.

Estimation of phytomass at the polygon level. For a typical ecoregion (No 2501 situated in middle taiga subzone of Irkutsk oblast'), the uncertainty of the total phytomass varies between $\pm 6 \%$ and $\pm 14 \%$ (mean $12 \%$ ). The range of uncertainty is similar for all forest fractions $( \pm 13-20 \%)$ apart from understory and green forest floor which have lower mean uncertainties, and foliage, which has a higher upper limit $( \pm 21-25 \%)$. The size of the $90 \%$ confidence interval normalized by area ranges between 0.67 and $31.60 \mathrm{Mg} \mathrm{C} \mathrm{ha}^{-1}$ (mean 13.44). The spatial distribution of the uncertainties is presented in Figure 1. As a whole, there are no spatial trends of the magnitude of uncertainty. Confidence intervals are mostly influenced by the average density of total phytomass by unit area.

Estimation of NPP at the polygon level. NPP of three aggregated fractions (above ground wood, green parts and below ground wood) was considered. The range of uncertainty is similar for each forest fraction, from less than $\pm 1 \%$ to between \pm 12 and $14 \%$. The mean values are $\pm 12 \%$ for the tree fractions and $\pm 8-9 \%$ for understory and green forest floor. Percentiles of 5 and $95 \%$ boundaries are similar for the above mentioned fractions, on average they are 0.92 and 1.08 of the mean, respectively. 
Estimation of NEP. Estimation of uncertainties of assessment of NEP has been done at the polygon level for each ecoregion and for the region of SIBERIA-II as a whole. The normalized range of NEP in ecoregion 2501 varies between 0.17 and $0.85 \mathrm{Mg} \mathrm{C} \mathrm{ha}^{-1}$ (mean of $0.67 \mathrm{Mg} \mathrm{C} \mathrm{ha}{ }^{-1}$ ). The normalized range of NEP varies across the Siberia-II region between 0.01 and $2.64 \mathrm{MgC} \mathrm{ha}^{-1}$ (mean of $0.51 \mathrm{Mg} \mathrm{C} \mathrm{ha}^{-1}$ ). There is a clear spatial trend in distribution of uncertainties across the region's area (Figure 2) what is explained by the increasing human impact on ecosystems from north to south.
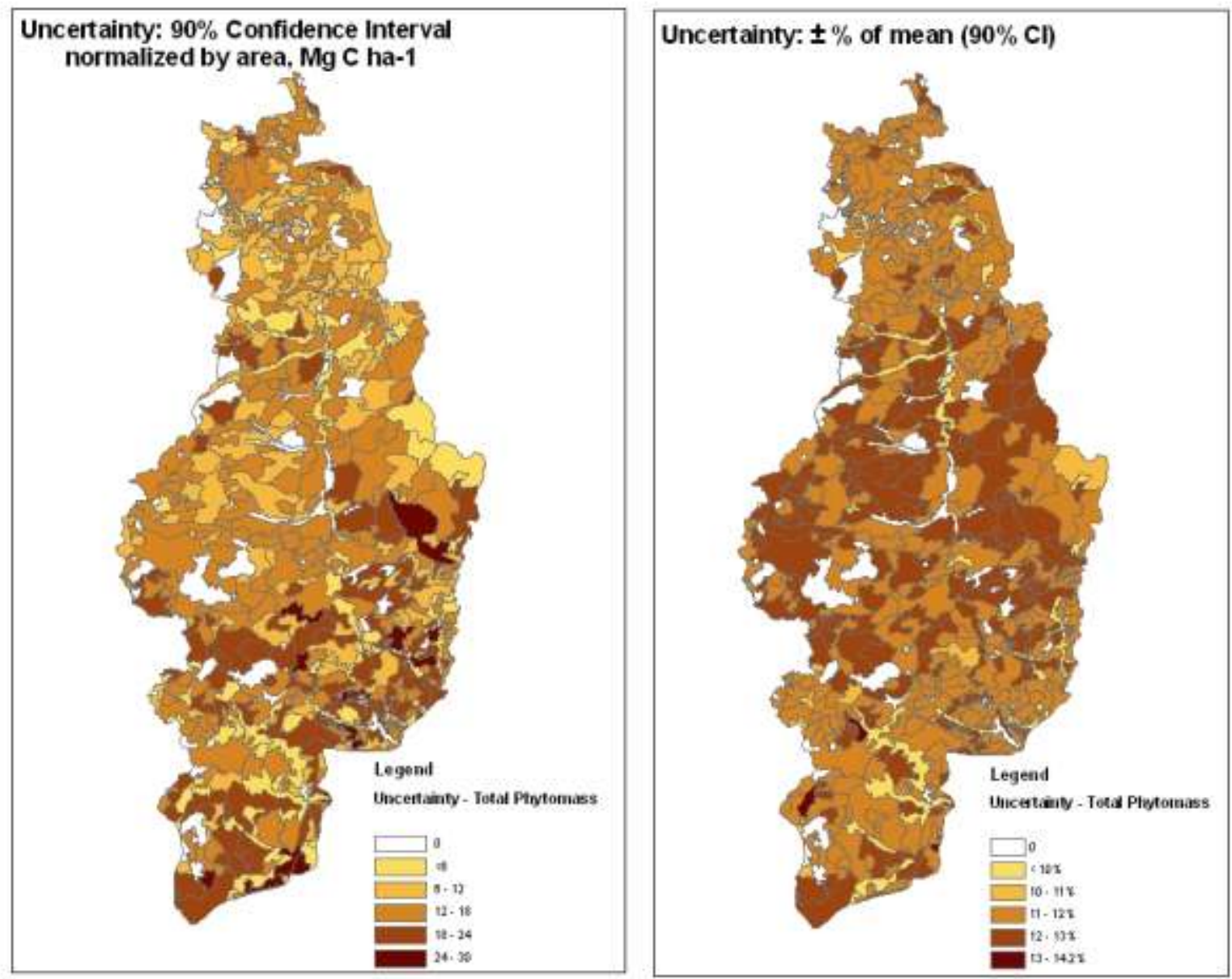

Figure 1. Uncertainties of total phytomass by polygon for ecoregion 2501 . The location of the ecoregion in the SIBERIA-II region is shown in Figure 2.

\section{End of Box 2 (the Box includes Figure 2)}

Different ways are possible to manage uncertainties based on additional information. There are many ways to evaluate the value of information, and most of them rely on determining the benefit of making a decision based on current knowledge versus spending more resources to improve the knowledge base that could be used in Bayesian decision analysis (Berger, 1985), 
or by referring to the more familiar expected value of perfect information (Morgan and Henrion, 1990). Effective ways of reducing carbon flux uncertainties strictly depend on the structure and specifics of the accounting schemes, and the most relevant ways to reduce their uncertainties differ from those required to reduce uncertainties in inventories of carbon pools. As a rule, an optimal way to reduce uncertainty requires a systems approach and lies in the attempt to utilize the synergism of combining heterogeneous information sources. For example, to substantially reduce the uncertainties of emissions caused by vegetation fires require more appropriate classifications (types of fires, types of combustibles, etc.) than are used in many countries, more accurate vegetation fuel maps, new or modified RS sensors, which enables identifying types of fire and their severity, and improved empirical models (e.g., to assess the amount of consumed combustibles of definite forest types depending on environmental indicators, fuel storage, etc.). In addition, it is necessary to keep in mind that some uncertainties cannot be reduced given current knowledge and economic conditions. 


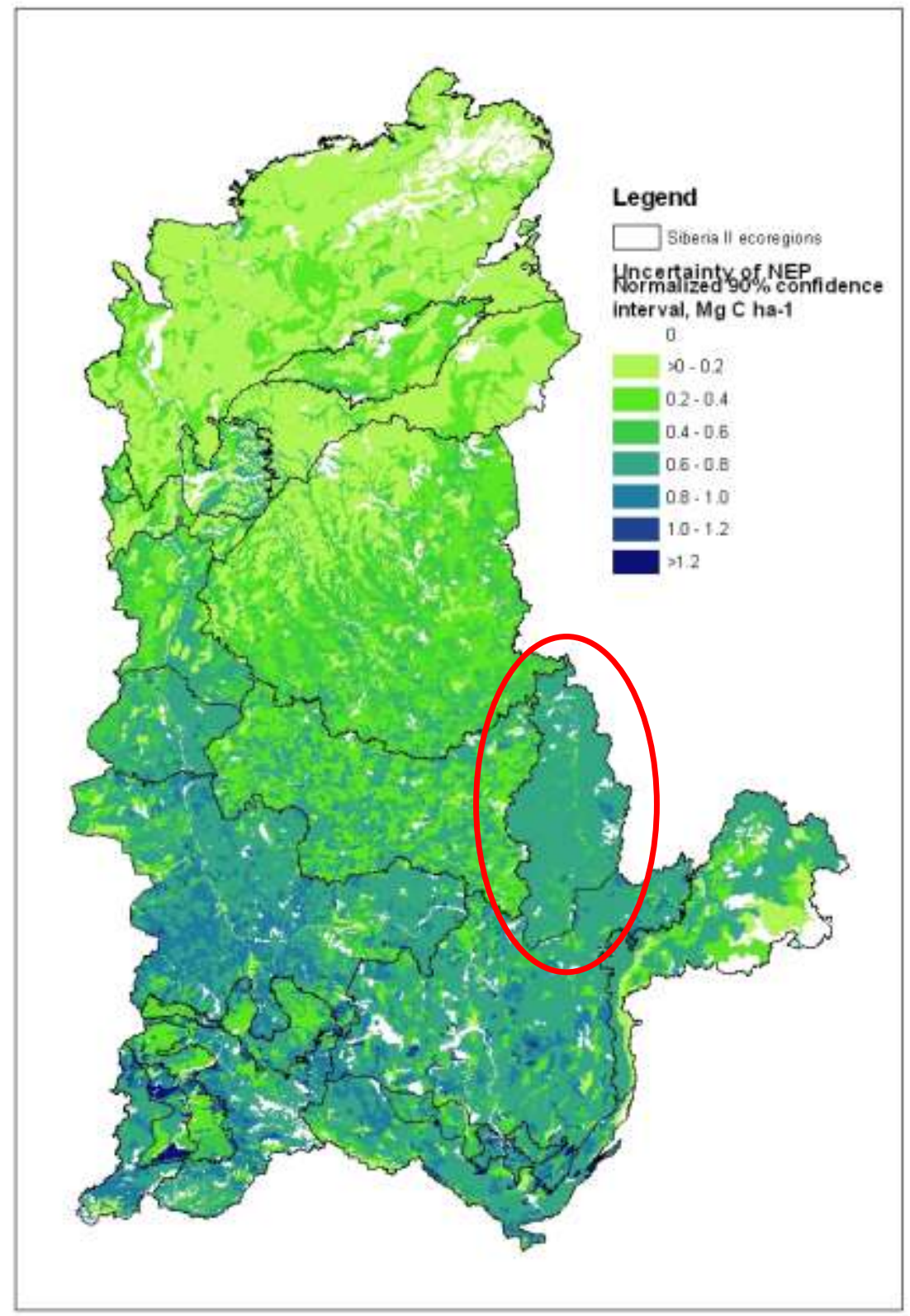

Figure 2. Uncertainty in NEP for all vegetation classes across the Siberia II region (uncertainty is shown as the $90 \%$ confidence interval normalized by polygon area). Red ellipse identifies ecoregion 2501 depicted in Figure 1.

\section{Conclusion}

The development of global integrated observing systems is a major strategic idea to establish verified regional terrestrial biota full carbon accounts in the future. The integrated observing system is understood as a permanent tool to combine all relevant information sources (onground measurements, remotely sensed data and empirical knowledge) and models of 
different types linked to primary polygons relevant for the FCA. Some prototypes of components of such systems and possible decisions are outlined above. Presumably, such an approach would allow to decrease uncertainties of annual NBP at regional and national scales to a range of 7 to $10 \%$. However, any proper development and implementation of such a system will require advanced theoretical and technical improvements, and development of new elements and subsystems. These improvements are mostly dealing with remote sensing, studying some poorly understood basic processes and developments of regional models of new types. Remotely sensed data are vitally important for the FCA. However, (1) only a multi-sensor remote sensing concept is able to satisfy major requirements of the accounting and (2) there is an evident need in development of new sensors which would be specifically addressed for assessing basic components of the FCA. One of substantial bottlenecks of the FCA is insufficient knowledge of ecosystem below ground processes. From the modeling point of view, it is clear that results produced by DGVMs for individual countries and continents have little in common with reality and their uncertainties still cannot be estimated in any formal way. Inventory-based modeling schemes are able to present only average data for some (rather uncertain) period. Recent developments show that "regionalizing" of DGVMs is a perspective way to introduce such models in verified FCA (Beer et al., 2006). In addition, there are promising results of the introduction of process-based elements in inventory-based approaches and how it was shown by the project SIBERIA-II. These can be considered as steps for developing new types of hybrid regional models which would keep the strengths and minimize weaknesses of both inventory and process based approaches.

An important unresolved question is setting the thresholds of relevant uncertainties which should be provided by verified regional and national FCA. There is no substantial progress in this field yet.

In the foreseeable future, the FCA will remain a fuzzy system in the sense which we discussed above. This implies that judgments about the reliability of the FCA will be based on a combination of strict formal methods as well as expert conclusions. In February 2005 the Kyoto Protocol has entered into force, and the technical task of assessing uncertainties has gained political and economic importance. This will require elaboration of the theoretical and practical aspects of the problem, as well as the development of special institutions, which would be responsible for certifying FCAs.

\section{References}

Alexeyev, V.A. and R.A. Birdsey (eds.), Carbon in Ecosystems of Forests and Wetlands of Russia. Sukachev Institute of Forest, Krasnoyarsk, 170 pp. [in Russian]. 
Bare, B.B. and G.A. Mendoza (1991), Timber harvest scheduling in a fuzzy decision environment. Canadian Journal of Forest Research 22, 423-428.

Beer C., W.Lucht, C. Schmullius and A. Shvidenko (2006). Uptake of carbon dioxide by forests in Russia. Geophysical Research Letters (in press).

Berger, J. (1985), Statistical Decision Theory \& Bayesian Analysis, $2^{\text {nd }}$ ed., John Wiley \& Sons, New York, 1984.

Cess, R.D., M.-H Zhang, G.L. Potter, et al. (1993), Uncertainties in carbon dioxide radiative forcing in atmospheric General Circulation Models. Science, Vol. 262, 1252-1255.

Chen, Q. and A.E. Mynett (2003), Integration of data mining techniques and heuristic knowledge in fuzzy logic modeling of eutrophication in Taihu Lake. Ecological Modelling 162, Issues 1-2, 55-67

Cogan, B. (2001), Certainty and Uncertainty in Science. Scientific Computing World, December, 2830.

Collins W.D., V. Ramaswamy, M.D. Schwarzkopf, et al. (2005), Radiative forcing by well mixed greenhouse gases: Estimates from climate models in the IPCC AR 4. Journal of Geophysical Research (in press). Available at http://www.cgd.ucar.edu/cms/wcollins/papers/

EEA (2005), Annual European Community Greenhouse Gas Inventory 1990-2003 and Inventory Report 2005. Submission o the UNFCCC Secretariat. Revised final version, 27 May 2005. European Environment Agency, Technical Report 4/2005, 241 pp.

FAO (2002-2005), Proceedings. Expert Meetings on Harmonizing Forest-Related Definitions for Use by Different Stakeholders. The First Meeting, 23-25 January 2002 (Rome, 2002, 193 pp.); the Second Meeting,11-13 September 2002 (Rome, 2002, 354 pp.); the Third Meeting, 17-10 January 2005 (Rome, 2005, 137 pp.).

GGP (2003), Global Carbon Project (2003) Science Framework and Implementation. Earth System Science Partnership (IGBP, IHDP, WCRP, DIVERSITAS) Report No.1; Global Carbon Project Report No.1, 69 pp, Canberra.

Haimes, Y.Y., T. Barry, and J.H. Lambert (1994), Workshop Proceedings: Where and how can you specify a probability distribution when you don't know much? Risk Analysis, Vol. 14, No. 5, 661-706.

Hattis, D., D.E. Burmaster (1994), Assessment of variability and uncertainty distributions for practical risk analysis. Risk Analysis 14, 713-730.

Heath, L.S., J.E. Smith (2000), An assessment of uncertainty in forest carbon budget projections. Environmental Science and Policy 3, 73-82.

Hofman F.O. and J.S. Hammonds (1994) Propagation of uncertainty in risk assessments: the need to distinguish between uncertainty due to lack of knowledge and uncertainty due to variability. Risk Analysis 14, 707-712.

IPCC (1997), Revised 1996 IPCC Guidelines for National Greenhouse Gas Inventories. Volume 1: Greenhouse Gas Inventory Reporting Instructions; Volume 2: Greenhouse Gas Inventory Workbook; Volume 3: Greenhouse Gas Inventory Reference Manual. IPCC/OECD/IEA. Intergovernmental Panel on Climate Change (IPCC) Working Group I (WG I) Technical Support Unit, Bracknell, United Kingdom. Available on the Internet: http://www.ipcc-nggip.iges.or.jp/public/gl/invs1.htm.

IPCC (1998), Managing Uncertainty in National Greenhouse Gas Inventories. IPCC/OECD/IEA Programme on National Greenhouse Gas Inventories, Meeting Report, 13-15 October, Paris, France.

IPCC (2000), Good Practice Guidance and Uncertainty Management in National Greenhouse Gas Inventories. J. Penman, D. Kruger, I. Galbally, T. Hiraishi, B. Nyenzi, S. Emmanuel, L. Buendia, R. Hoppaus, T. Martinsen, J. Meijer, K. Miwa and K. Tanabe (eds.), Intergovernmental Panel on Climate Change (IPCC) National Gas Inventories Program, Technical Support Unit, Institute for Global Environmental Strategies, Hayama, Kanagawa, Japan.

IPCC (2004), Documents in Support of the Writing Process for the IPCC Working Group II Fourth Assessment Report. Volume produced for the first Lead Authors Meeting, Vienna, 20-23 September 2004.

IPCC (2004a), IPCC Workshop on Describing Scientific Uncertainties in Climate Change to Support Analysis of Risk and of Options, Maynooth, Co.Kildare, Ireland, 11-13 May 2004. Workshop Report 
edited by M. Manning, M. Petit, D. Easterling, J. Murphy, A. Patwardhan, H.-H. Rogner, R. Swart and G. Yohe. Available at http://ipcc-wg1.ucar.edu/meeting/URW/

Isaev A.S., G.N. Korovin, A.I. Utkin, A.A. Pryashnikov, and D.G. Zamolodchikov (1995), Carbon sock and deposition in phytomass of the Russian forests. Water, Air and Soil Pollution 70, 247-256

Isaev, A.S. and G.N. Korovin (1998), Carbon in forests of Northern Eurasia. In: G.A. Zavarzin (ed.) Carbon Turnover in Territories of Russia. Ministry of Science and Technology of the Russian Federation, Moscow, pp. 63-95 [in Russian].

Jonas, M., S. Nilsson, A. Shvidenko, V. Stolbovoi, M. Gluck, M. Obersteiner and A. Oeskog (1999), Full Carbon Accounting and the Kyoto Protocol: A Systems-Analytical View, Interim Report IR-99025, International Institute for Applied Systems Analysis, Laxenburg, Austria. Available at: http://www.iiasa.ac.at/Publications/Documents/IR-99-025.pdf

Kosko, B. (1994), Fuzzy Thinking, Flamengo, 245 pp.

Lapenis, A., A. Shidenko A., D. Sheschenko, S. Nilsson and A.Aiyyer (2005), Acclimation of Russian forests to recent changes in climate. Global Change Biology 11, 1-13, doi. 10.1111/j.13652486.2005.01069.x

MacFarlane, D.W. E.J. Green and H.T. Valentine (2000), Incorporating uncertainty into the parameters of a forest process model. Ecological Modelling, Issue 1, 27-40.

Mendoza, G.A. and W.L. Sprouse (1989), Forest planning and decision making under fuzzy environments: an overview and illustration. Forest Science 32, 481-502.

Monni, S., S. Syri and I. Savolainen (2004), Uncertainties in the Finnish greenhouse gas emission inventory, Environmental Science and Policy 7, 87-98.

Morgan, M.G. and M. Henrion (1990), Uncertainty: A Guide to Dealing with Uncertainty in Quantitative Risk and Policy Analysis, Cambridge University Press, New York.

Moss, R.H. and S.H Schneider (2000), Uncertainties in the IPCC TAR: Recommendations to lead authors for more consistent assessment and reporting. In: R. Pachauri, T. Taniguchi and K. Tanaka (eds.) Guidance Papers on the Cross Cutting Issues of the Third Assessment Report of the IPCC. Intergovernmental Panel on Climate Change, Geneva, Switzerland, 33-51.

Myneni, R.B., J. Dong, C.J. Tucker et al. (2001) A large carbon sink in the woody biomass of Northern forests. Proceedings of the National Academy of Sciences, USA 98(26), 1478414789.

National Assessment Synthesis Team (2001), Climate Change Impacts on the United States: The Potential Consequences of Climate Variability and Change, Report for the US Global Change Research Program, Cambridge University Press, Cambridge, UK, 620 pp.

Özesmi, U. and S.L. Özesmi (2004), Ecological models based on people's knowledge: a multi-step fuzzy cognitive mapping approach. Ecological Modelling 176, Issues 1-2, 43-64

Nilsson, S., A. Shvidenko, V. Stolbovoi, M. Gluck, M. Jonas and M. Obersteiner (2000a), Full Carbon Account for Russia. Interim Report IR-00-021. International Institute for Applied Systems Analysis, Laxenburg, Austria. Available at: http://www.iiasa.ac.at/Publications/Documents/IR-00-021.pdf, Study also featured in: New Scientist, 2253, 26 August 2000, 18-19.

Nilsson, S., M. Jonas and M. Obersteiner (2000b), The Forgotten Obligations in the Kyoto Negotiations. Document made available by the International Institute for Applied Systems Analysis, Laxenburg, Austria, on the Internet: http://www.iiasa.ac.at/Research/FOR/ carb_kyoto.html?sb=10.

Nilsson, S., M. Jonas, M. Obersteiner and D. Victor (2001), Verification: The Gorilla in the Struggle to Slow Global Warming. The Forestry Chronicle 77, 475-478.

Nilsson, S., E.A. Vaganov, V.A. Rozhkov, A.Z. Shvidenko, V.S. Stolbovoi, I. McCallum, M. Jonas and M. Obersteiner (2003a), Greenhouse Gas Balance and Mitigation Strategies for Russia, World Climate Conference, September 29 - October 3, 2003, Abstracts, Moscow, 242-243.

Nilsson, S., M. Jonas, V. Stolbovoi, A. Shvidenko, M. Obersteiner and I. McCallum (2003b), The Missing "Missing Sink", The Forestry Chronicle 79, No 6, 1071-1074. 
Parysow P., G. Gertner and J. Westervelt (2000), Efficient approximation for building error budgets for process models. Ecological Modelling 135, Issues 2-3, 111-125.

Rowe, W.D. (1994), Understanding Uncertainty. Risk Analysis 14, No 5, 743-750.

Rypdal, K.L., and W. Winiwarter (2001), Uncertainties in greenhouse gas inventories-evaluation, comparability and implications. Environmental Science Policy 4, 107-116.

Schulze, E.-D., R. Valentini and M.-J. Sanz (2002), The Long Way from Kyoto to Marrakesh: Implications of the Kyoto Protocol Negotiations for Global Ecology. Global Change Ecology 8, 505518.

Shvidenko A., S. Nilsson, V. Rojkov and V. Strakhov (1996) Carbon budget of the Russian boreal forests: a system analysis approach to uncertainty. In: M.J. Apps, D.T. Price (eds.) Forest Ecosystems, Forest Management and the Global Carbon Cycle, NATO ASI series, Ser.1, Vol.40, 145-162.

Shvidenko, A., D. Shepashenko, and S. Nilsson (2002), Aggregated models of phytomass of major forest forming species of Russia. Forest Inventory and Management, No 1, 50-57, Krasnoyarsk [in Russian]

Shvidenko, A. and S. Nilsson (2002), Dynamics of Russian forests and the carbon budget in 19611998: An assessment based on long-term forest inventory data. Climatic Change 55, 5-37.

Shvidenko, A., and S. Nilsson (2003), A Synthesis of the Impact of Russian Forests on the Global Carbon Budget for 1961-1968, Tellus 55B, 391-415.

Shvidenko, A., D.Shepashenko, S. Nilsson and Yu. Bouloui (2004), The system of models of biological productivity of Russian forests, Forestry and Forest Management, No 2, 40-44 [in Russian].

Shvidenko, A., D. Shepaschenko, S. Nilsson and E. Vaganov (2006), Dynamics of phytomass and Net Primary Production of Russian forests: New estimates. Doklady of the Russian Academy of Sciemces (submitted).

Steffen, W., I. Noble, J. Canadell, M. Apps, E.-D. Schulze, P.G. Jarvis, D. Baldocchi, P. Ciais, W. Cramer, J. Ehleringer, G. Farquhar, C.B. Field, A. Ghazi, R. Gifford, M. Heimann, R. Houghton, P. Kabat, C. Körner, E. Lambin, S. Linder, H.A. Mooney, D. Murdiyarso, W.M. Post, I.C. Prentice, M.R. Raupach, D.S. Schimel, A. Shvidenko and R. Valentini (1998), The Terrestrial Carbon Cycle: Implications for the Kyoto Protocol. Science 280, 1393-1394.

Vasiliev, S.V., A.A. Titlyanova and A.A. Velichko (eds.) (2001), West Siberian Peatlands and Carbon Cycle: Past and Present. Proceedings of the International Symposium, Noyabrsk, August 18-22, 2001. Novosibirsk, RAS, $250 \mathrm{pp}$.

Wang, Y.P. and D.J. Barret (2003) Estimating regional terrestrial carbon fluxes for the Australia continent using a multiple-constraint approach: 1. Using remotely sensed data and ecological observation ns of net primary production. Tellus 55B, 270-279.

Wan-Xiong, W., L. Yi-Min, L. Zi-Zhen and Y. Fengxiang (2003), A fuzzy description on some ecological concept. Ecological Modelling 169, Issues 2-3, 361-366.

Zadeh, L. (1965), Fuzzy sets. Inf. Control 8: 338-353.

Zaehle, S., S.Sitch, B. Smith and F. Hatterman (2005), Effects of parameter uncertainties on the modeling of terrestrial biosphere dynamics. Global Biogeochemical Cycles 19, GB3020, doi: $10.1029 / 2004$ GB002395.

Acknowledgement: The SIBERIA-II project (EVG-2001-00008), 2003-2005, is funded by the European Commission (Generic Activity 7.2: Development of Generic Earth Observation Technologies) 